\title{
Individual learning path personalization approach in a virtual learning environment according to the dynamically changing learning styles and knowledge levels of the learner
}

\author{
T. M. A. U. Gunathilaka ${ }^{1,}{ }^{*}$, M. S. D. Fernando ${ }^{2}$, H. Pasqual ${ }^{3}$ \\ ${ }^{1}$ Department of Physical Sciences, Rajarata University of Sri Lanka, Mihintale, Sri Lanka \\ 2 Department of Computer Science and Engineering, University of Moratuwa, Moratuwa, Sri Lanka \\ ${ }^{3}$ Department of Electrical and Computer Engineering, Open University of Sri Lanka, Colombo, Sri Lanka
}

\section{A R T I C L E IN F O}

\section{Article history:}

Received 6 December 2017

Received in revised form

20 February 2018

Accepted 1 March 2018

\section{Keywords:}

Learning behaviour

Learning personalization

Objective Setting

Learning styles

Learning path

\begin{abstract}
A B S T R A C T
The Traditional pedagogical approaches to learning are mostly instructorcentered. Since the students or the learners are individually in different knowledge levels, often, they are unable to obtain the effective usage of the teaching methods to improve their knowledge alike. Although the interactive mechanisms are presented with modern e-learning solutions, mechanisms on paying concentration on the delivery of learning materials targeting on each individual student separately for equal knowledge distribution are very rare. As a solution to distribute the learning process in a way to obtain the knowledge by the students equally, this research is carried out to personalize the learning material delivery among the individual students according to their own static and dynamic learning behaviors and the dynamically changing knowledge levels. Such styles are extracted through a literature base study and the analyzed learning behaviors obtained through students' login profiles in a Learning Management System. In the related studies, they have pointed out some personalizing approaches related to several models of learning theories and the learning styles. Further, with the mobile apps developed for Educational purposes, they have done personalization up to a certain level by considering their accessing history of the educational content. In such approaches, although they have statistically measured the students in numbers to divide them for their learning styles, there is no any mathematical model presented to accurately identify such styles. The main objective of this research is to recursively personalize the learning path according to the dynamically changing learning styles of the student according to an implemented mathematical model for continuous delivery of learning materials and evaluation of their performance until the expected lesson objectives are satisfied by the student. Then based on the identified individual styles, learning path personalization is done as a tree traversal approach in the lesson plan which is delivered as an n-ary tree data structure. By delivering this personalization approach by implementing it in a Virtual Learning Environment (VLE), it was able to obtain more than $70 \%$ of learning performance enhancement within individual students with several undergraduate course units.
\end{abstract}

(C) 2018 The Authors. Published by IASE. This is an open access article under the CC BY-NC-ND license (http://creativecommons.org/licenses/by-nc-nd/4.0/).

\section{Introduction}

According to the background study, I have identified that there are multiple e learning solutions to facilitate the students with distance learning (Mukherjee, 2013; Bauman and Tuzhilin, 2016).

\footnotetext{
* Corresponding Author.

Email Address: anupama929@as.rjt.ac.lk (T. M. A. U. Gunathilaka) https://doi.org/10.21833/ijaas.2018.05.002

2313-626X/C) 2018 The Authors. Published by IASE.

This is an open access article under the CC BY-NC-ND license

(http://creativecommons.org/licenses/by-nc-nd/4.0/)
}

With the advanced capabilities and interactive mechanisms available with mobile devices, they have been effectively used in delivering the learning contents and teaching methods. The problem currently that is being faced by the students is that the inabilities of getting and understanding the teacher's lesson contents delivery to enhance individual knowledge alike, although there are such advance technologies exists in such environments.

On the other hand, although the lesson planning strategies according to the modern learning methods are presented in the education system such as 
SCORM standards (ADLS, 2011), teachers who are following them practically are somewhat rare specially within e learning environments. The reasons mentioned by them for their failure are the less interactivity, the less awareness on the way of applying them in the VLEs and the less students motivation in actual learning since all of them are attending to the coursework with the intention of only passing the exams where most of them are not success throughout the course with objective based learning. Therefore, the teachers' preference is on a personalized learning environment where the outcome based learning can be offered with delivering lessons through individual's preferred ways of learning to motivate them in the education process.

Therefore, finally I came up with a research problem, " there is no a proper and interactive way of personalizing the learning path of the individual students according to their dynamically changing learning behaviors and the preferences in an objective based course structures where at the same time which can be implemented in the same learning environment"

Further in this research work, the objective is to priorities the learning process according to the individual students learning behavior and the knowledge level while facilitating the teacher to guide them through an objective basis lesson plan.

Initially, according to the identified models of learning styles, a selected sample of students is categorized based on their static and dynamic learning behaviors to focus on personalization (Gunathilaka et al., 2017). The survey is carried out with 2 different data sets: Learning Management System (LMS) logging information profiles of the students and the data set collected on a questionnaire, distributed among the same student group. Basically from the questionnaire, the static learning behaviors such as the preferred way of learning object representation strategies, their field of expertise etc. are obtained. From the behaviors in the LMS, the dynamically changing learning behaviors on a particular lesson are collected. Student sample, from the population who are following the subjects of Management and Information Systems (MIS), Data Structures and Algorithms and Control System Engineering in a Computer Engineering Degree program are selected to observe the learning behaviors.

The questionnaire which is prepared according to the Multiple Intelligence model and tests experimented by Howard Garner has been used to collect the static learning preferences of the learner which are hard to find out through LMS logging profiles.

As the second approach, outcome basis course taxonomy (Bauman and Tuzhilin, 2016) has been implemented as a hierarchy which guides the user towards successful achievement of the course objectives according to the expected level of knowledge. The learner is guided through different learning paths according to their preferences and the current level of the knowledge extracted, until he reaches the expected knowledge level of a certain objective. The personalization structure through the objective based course taxonomy has been organized as an $\mathrm{N}$-ary tree (Lafore, 2002) data structure which recursively changes the learning paths of the students when they traverse across it.

From the entire research, it has been focused towards automatic detection of the learning style and the preference and delivering the common learning materials by customizing them according to the individual's preferences as a learning path.

\section{Approach of learning behavior identification}

According to Kolb's learning style model, learning styles of individuals are based on 4 stages on the learning process (McLeod, 2013). By observing the pattern of learning through these stages, Kolb has proposed that there are four types of learning styles exist among the students such as diverging, assimilating, converging, and accommodating. From Howard Garner's multiple intelligences model, it can obtain the students' intelligence areas such as Linguistic, music, body and kinesthetic, logical and mathematical intelligence, which will lead to their preferred way of learning. The Visual Audio Kinesthetic (VAK) model provides a different perspective for understanding and explaining a person's preferred or dominant thinking and learning style with audio, visual and kinesthetic means. In Felder Silverman model, it describes each person's preferred way of perceiving information, information representation, processing and organizing the same for understanding and making conclusions (Graf et al., 2007).

In this research, according to the identified learning styles at each stage of learning, the students are categorized with the analyzed behavioral and preference components, collected through the survey carried out with LMS and the questionnaire.

The questionnaire which is prepared to collect the static learning behaviors of students has been modeled according to Multiple Intelligence Model and VAK model. The main focus of the questionnaire is to collect such preferences, since it is hard to collect the static preferences through an e learning environment such as LMS.

120 number of students group is selected from the previously mentioned population. Different types of activities and the various student behaviors on those activities have been considered for determining the specific learning style. It is done by a rule base. By the questionnaire results, preferred intelligence is identified to verify and add additional parameters for the same set of rules to determine student learning preferences.

\subsection{Questionnaire for static behavior extraction}

As the static behaviors, preferred way of the education material presentation strategy, common interested fields of studies of the individual students 
have been identified. In here, the interests are taken in general not as subject specific factors. The VAK model provides a different perspective for understanding and explaining a person's preferred or dominant thinking, learning style, and strengths. According to VAK model, it has demonstrated that there are 3 ways the students prefer learning. They are based on the learning by visual form, auditory form and the kinesthetic means.

According to Howard Garner's Multiple Intelligence Model, seven Multiple Intelligences are being suggested to explain people's preferred ways to learn and develop. According to Garner, the types of intelligence that a person possesses indicate not only a person's capabilities, but also the manner or method in which they prefer to learn and develop their strengths. By Garner, he has conducted multiple intelligence tests to demonstrate his model.
Further this questionnaire has been prepared by following those tests to gather their preferred ways of learning. Questionnaire has general questions from which I have extracted their presentation preferences and common interests. The questionnaire doesn't contain the questions related to subject matters since such types of questions will be biased towards a particular lesson/course. Further, from each discipline, multiple questions have been asked to avoid straight forwardness from a single question. It is a MCQ type questionnaire, where for each question is given set of answers. Each question on the questionnaire has been targeted on a particular category of preference.

Every answer is given a particular ranking as in Table 1 according to its biasness towards a particular preferences/style according to the question.

Table 1: Rakings given for answers in questionnaire

\begin{tabular}{|c|c|c|c|c|c|c|c|c|c|c|c|}
\hline \multirow{2}{*}{$\begin{array}{l}\text { Question } \\
\text { Number }\end{array}$} & \multicolumn{4}{|c|}{ Presentation preference } & \multicolumn{3}{|c|}{ Interest } & \multicolumn{4}{|c|}{ Style } \\
\hline & Kinesthetic & Audio & Visual & Reading & Numerical & Aesthetic & History & Accommodating & Diverging & Assimilating & Converging \\
\hline 1 & 3 & & & & & & & 3 & & & 2 \\
\hline 2 & & 3 & & & & & & & 2 & 1 & \\
\hline 3 & & & 3 & & & & & & 2 & 1 & \\
\hline 4 & & & & 3 & & & & 2 & & 1 & \\
\hline 5 & & & & & 3 & & & & & & 3 \\
\hline 6 & & & & & & 3 & & & & & \\
\hline 7 & & & & & & & 3 & & & & \\
\hline 8 & & & & & & & & 1 & & 3 & \\
\hline 9 & & & & & & & & & 3 & & \\
\hline 10 & & & & & & & & 1 & & 2 & 1 \\
\hline
\end{tabular}

Finally with the student answer sheet on the questionnaire, with the ranks assigned, their most preferred ways of learning material representation strategy, their interesting field of study are calculated. Eqs. 1 and 2 which are mentioned below shows how the most preferred learning style of the student is calculated. According to Felder Silverman model (Graf et al., 2007) it has identified the learning styles and the individual's preferences on each style based on the stage of learning.

$l s s q=\sum_{i=1}^{n}\left(r_{i} \cdot a_{i}\right)$

lssq - learning style score obtained from questionnaire

$r$ - Rates assigned for an answer

$a$ - status of the answer (if answered $\mathrm{a}=1$ else $\mathrm{a}=0$ )

$i$ - Each answer under a learning style

$n$ - Number of answers under a learning style

$s l b=\operatorname{Max}(l s s q)(2)$

$s l b$ - student learning behavior

\subsection{Dynamic behavior of learning}

The other way I have identified the student learning behavior is by analyzing the dynamically changing behaviors of learning collected based on their LMS logging profiles. For the analysis of dynamic behaviors of learning, 120 numbers of students group is selected from the above mentioned population to observe their behaviors in the LMS. Types of activities selected from the LMS are forums, assignments, practical, quizzes, lecture notes, web resources etc. Average participation on each activity components, frequency of participation on each activity, precedence of accessing activities, time spent on each activity, activities skipped, how often they have logged in to LMS to access activities, number of forum posts and grades obtained, contribution to group works etc. have been considered to determine the parameters for learning style identification rules.

Then a rule set has been introduced to categories their preferred way of learning according to the analyzed behaviors as mentioned above. As an example, if a student has accessed $70 \%$ of the forums from the available forums, the rule determine that person is interested in forum activities. After determining these preferences, each activity preference same as in identification of the static behavior, is given rating based on their contribution towards a particular learning style. Rates associated with the preferences of the activities are mentioned in Table 2. The learning styles are determined through the study of Felder Silverman model and Kolb's model. In Kolb's model (McLeod, 2013) it describes learning styles of individuals are derived on 4 stages on the learning cycle. He suggests by transmitting through pairs of these stages, students show different learning styles. Learning styles that have been identified by Kolb (McLeod, 2013) based on such stages are:

- Diverging 
- Assimilating

- Converging

- Accommodating

According to Kolb (McLeod, 2013), people with diverging learning style prefer feel and watch. They are preferred in gathering information, use imaginations to solve problems, like the people with same thinking and like group working etc. People with assimilating style prefer watching and thinking, more interested in ideas and abstract concepts than people and practical situations. People with this style prefer readings, lectures, exploring analytical models, and having time to think things through. Then who have converging style, like doing and thinking. They use their learning to find solutions to practical issues. They prefer technical tasks, and are less concerned with people and interpersonal aspects. People with a Converging learning style are best at finding practical uses for ideas and theories. They are preferred in experimenting with new ideas, to simulate, and to work with practical applications. Accommodating style is with the persons preferred doing and feeling. Accommodating learning style prefer to work in teams to complete tasks. They set targets and actively work in the field trying different ways to achieve an objective and they use others analysis only to take information. They use their own mind to do creative works (McLeod, 2013).

Learning styles according the Felder Silverman (Graf et al., 2007):

- Sensitive/intuitive - continuum determines how people are likely to take and perceive information.

- Visual/ audio- determine how the people are likely the information to be presented.

- Active/reflective - determine how they prefer to process information.

- Sequential / global- determine how the people prefer to organize and progress towards understanding info.

In the survey, I have categorized the learning contents of the courses based on the stage of the delivery through lessons within the semester. The stages are knowledge acquiring stage, processing stage and understanding stage. For each student, their interested way of learning is determined based on their behavior at each stage to dynamically identify whether their learning behavior is changed in different levels/stages of the learning process. Based on the similarities exists within Silverman and Kolb's model (Graf et al., 2007; McLeod, 2013) it is able to group each styles in to several categories.

Table 2: Rakings given for LMS activity preferences

\begin{tabular}{|c|c|c|c|c|c|c|c|c|c|c|}
\hline Interest & accommodating & converging & diverging & assimilating & reflective & active & sensitive & intuitive & Sequential & Global \\
\hline Like forums & 2 & & 3 & 1 & & 2 & & & & \\
\hline $\begin{array}{l}\text { Like group } \\
\text { works }\end{array}$ & 3 & & 2 & 3 & & 3 & & & & \\
\hline $\begin{array}{c}\text { Like extra } \\
\text { knowledge }\end{array}$ & 1 & & 2 & 3 & 3 & & & & & \\
\hline $\begin{array}{l}\text { Like } \\
\text { assignments }\end{array}$ & 2 & 3 & & & & & 3 & 3 & & \\
\hline $\begin{array}{l}\text { Like teacher } \\
\text { notes }\end{array}$ & & & 2 & 3 & 3 & & & & & \\
\hline $\begin{array}{l}\text { Like practical } \\
\text { knowledge }\end{array}$ & 2 & 3 & & & & & 3 & & & \\
\hline p-s & & & & 3 & 3 & & & & & \\
\hline l-s & 2 & 3 & & & & & 3 & 3 & & \\
\hline order & & & & & & & & & 3 & \\
\hline Not in order & & & & & & & & & & 3 \\
\hline
\end{tabular}

Based on their likeliness towards different types of methods of learning which are always dynamically changed, their most preferred dynamic learning styles have been obtained. For each student, behaviors in the LMS are considered to determine their dynamic learning behavior according to the Eqs. 3 and 4.

$$
l s s=\sum_{i=1}^{n}\left(r_{i} \cdot l_{i}\right)
$$

lss-learning style score

$r$ - rates assigned for activity preference

$l$ - likeliness(if like $\mathrm{l}=1$ else $\mathrm{l}=0$ )

$i$ - each preferred activity under a learning style

$n$ - number of preferred activities under a learning style

$s l b=\operatorname{Max}(l s s)(4)$

$s l b$ - student learning behaviour
Likewise, for static behavior and dynamic behavior (Murray and Pérez, 2015), I have assigned priorities for their behavior which is finally discovered, from most preferred way to least preferred way. The main target of prioritizing the order is, when preparing the learning material, if it cannot be customized to every style; deliver a material which is similar to the second prioritized style based on the possibility. On the other hand if the student is not being satisfied a lesson objective expected from a particular activity which is prepared and delivered according to their highest priority style, by navigating them through another path based on their next priority level, getting them to the desired level is also been expected here.

\section{Pedagogy for learning path personalization}

As stated in the previous explanation, the research objective was to develop a personalized 
learning environment for education performance enhancement of the students. For personalization, I have considered the static and dynamic learning behaviors of the students. Further a new parameter is added which is the current knowledge level of the student in a particular lesson. Other than the knowledge level at an intermediate position in a lesson, prior to deliver a particular lesson, it is needed to understand whether the learner is in a position/level to understand the material which is described as the readiness. It's basically the initial knowledge level of the student before the lesson. I have done it by delivering them an activity first. The teacher should have to develop an activity prior to the material delivery which is composed of the prerequisite knowledge which is needed to attend to the activity and understand the knowledge level of the student. Based on the level, the student is navigated through a learning path which is personalized with their individual learning styles identified. The lesson structure has been organized as a hierarchy (Bauman and Tuzhilin, 2016) which guides the user towards achieving the learning outcomes. Likewise, according to the stages of learning, specifically at the knowledge acquiring stage and processing stage, individual knowledge levels are measured with the formative assessments (Mukherjee, 2013). To fill the gap between the current knowledge level and the expected knowledge level, student learning path is always generated according to their learning styles.

\section{Lesson planning}

In general, when planning a lesson or a course, the initial fact to be considered is objective setting. At the end of the lesson, the teacher's aim is to measure whether the students are satisfied with the specified objectives to an expected level by continuing through the lesson. The lesson is composed of several topics, where each of those topics contributes in achieving specific objective/objectives by forming a hierarchy.

Hierarchy has 2 parts, course taxonomy and personalization as shown in Fig. 1. Within one part, each level specifies different aspect. Means all the nodes are not same. It forms an N-ray data structure (Lafore, 2002). But siblings are in same category. Lesson has been divided in to topics and sub topics. For a specific lesson objective to be achieved, student should have to come to a certain knowledge level in the associated topics under a particular objective. Here at the topic level, it has assigned a specific rank for a topic (ex: based on each topic's amount of contribution to the objective achievement as mandatory, desired, and better to follow)

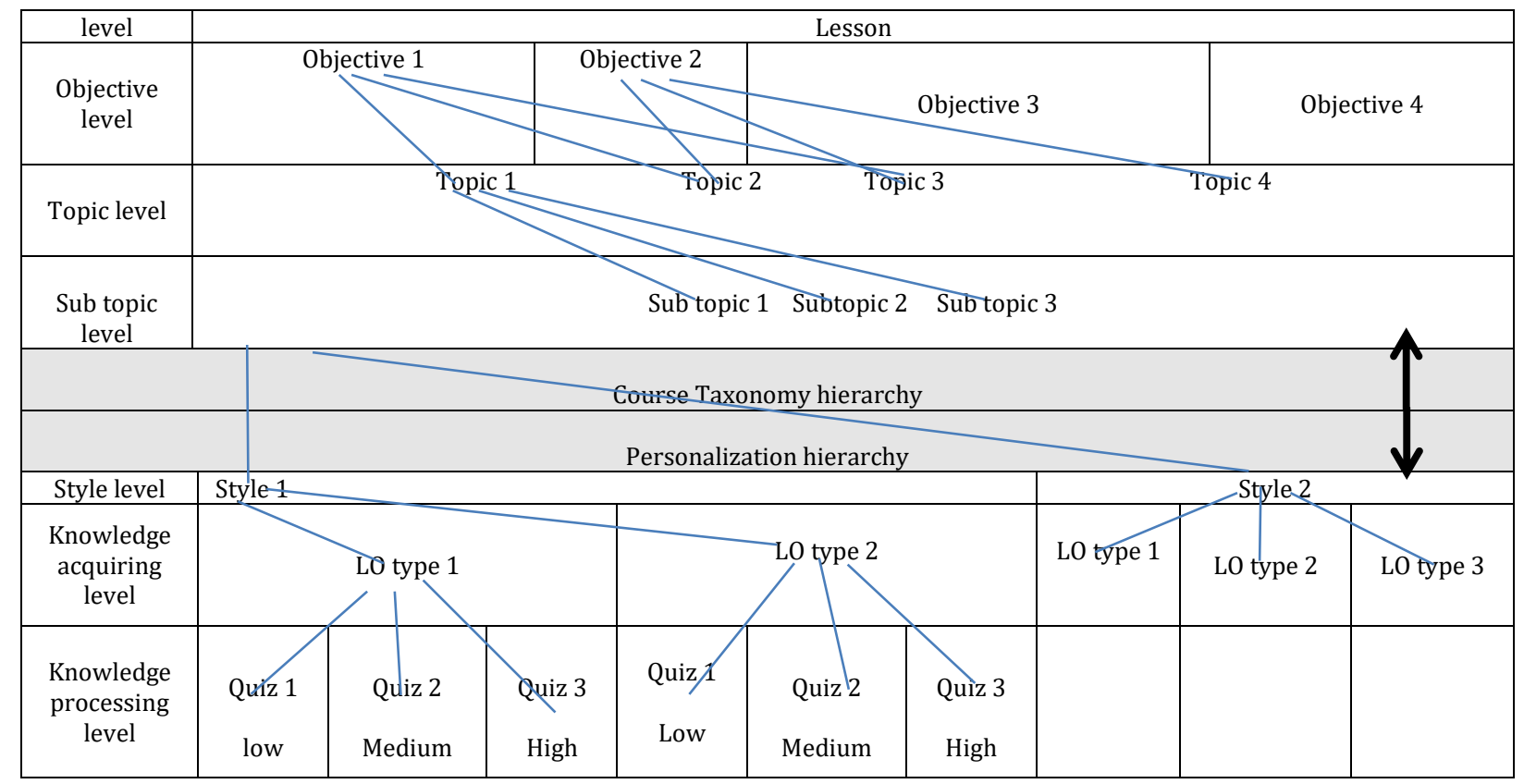

Fig. 1: Taxonomy of objective based lesson plan

At sub topic level the same thing has been done, based on each sub topics' contribution to the topic. Main aim is to take the student to a specific higher knowledge level from the current knowledge level. This is where done it through personalization. Likewise, from top to bottom of the hierarchy of the course taxonomy, immediate child level, child nodes individually contribute their values to fulfill the associated parents' target knowledge level with a particular amount. From each level, student should have to come to the expected level to proceed further. Associations among lesson components are shown in Fig. 2.

\section{Personalization of the learning path}

In this research, course materials are developed by targeting a specific objective in the lesson. Each material has multiple objects customized according to different learning styles identified. These 
materials are also differed according to the stages they are delivered.

First, the knowledge acquiring stage, where the students are grabbing the knowledge from the lesson contents, the lesson materials are provided according to the style identified. Initially under each sub topic, the lesson contents/learning objects are delivered in various ways by targeting at different styles of the students. For a specific style also there are several activity types. At the knowledge acquiring level, from one specific type according to the calculated learning style of the student (which is obtained through the Eqs. 1-4 knowledge is delivered. Then they are processing the knowledge based on the knowledge acquired. The teacher is able to observe the student knowledge processing through formative assessments (Mukherjee, 2013). From these formative assessments, delivered according to the students' style at the moment, their knowledge level is identified at each level.

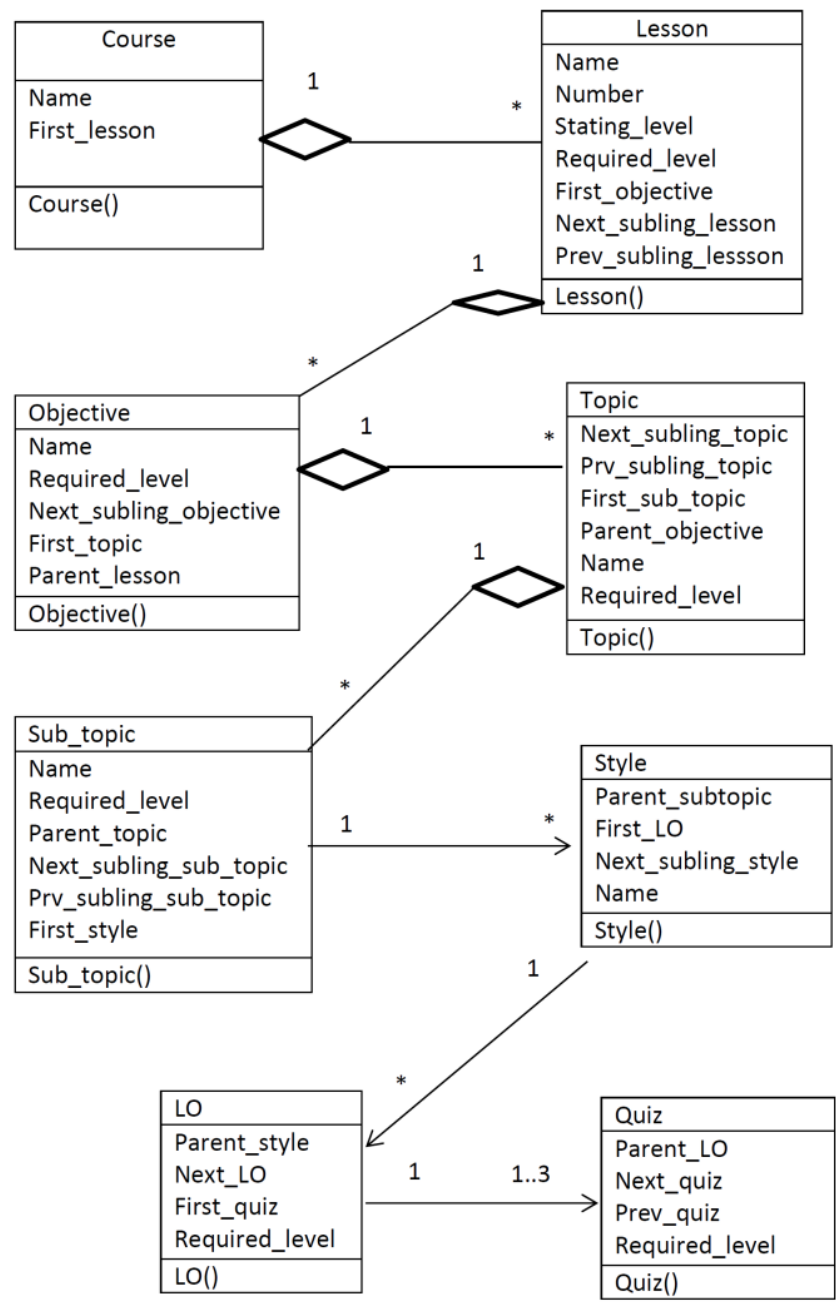

Fig. 2: Associations between lesson components

Then to identify whether they have acquired the knowledge desired, under each type delivery, it will give 3 assessments with complexity levels low, medium and high. Initially assessment with low complexity is offered. If the student has succeeded in the 1st one (means student is in the desired level in basic knowledge- calculated based on a mark range) and then will be navigated to the assessment with medium complexity in the same set (ex: under Learning Object/LO type 1) etc. If a student is failed with a specific level assessment on a particular LO type (suppose at medium quiz) he will be reinforced to the next LO type for improving some knowledge on same sub topic further and transferred to the assessment with medium complexity under that LO type. Likewise for each failing point they will be navigated to the next LO type for knowledge enhancement and transferred to the assessment with the complexity level where it has failed on the previous one.

For each sub topic contribution for a topic is mandatory. High overall performance in the quiz is desired. If it is sufficient to have moderate performance, medium performance is ok. But they are reinforced to complete the high level assessment as a desired option. If they are in a low level, finally they will be reinforced to follow quiz with medium level as mandatory. If the required level of the sub topic is mandatory, for the students medium and high level assessments are reinforced as mandatory to follow.

Main factor which is wanted to achieve here is initially allow them to acquire the knowledge with identified style (what we propose based on their 
calculated behavior) and then allow to process knowledge through the assessments. Then based on their performance level, if the required level is not met they will be navigated through the other knowledge acquiring objects within their style until a desired result is obtained.

Then the understanding of knowledge which can be identified by the practical applications the students do after the lesson can be monitored through summative assessments. This is specifically for evaluation purpose. Since all summative evaluation methods are common for all the students (not in the forms specific styles). Therefore at this stage all are required to face for the common tests and check whether they are enhanced with the knowledge as expected. Under the style level, the students learning styles are dynamically generated based on their behavior in the same learning environment as discussed in the analysis above. Based on the generated styles, their learning path is customized according to their preferred ways. Algorithm for personalization is shown in Fig. 3.
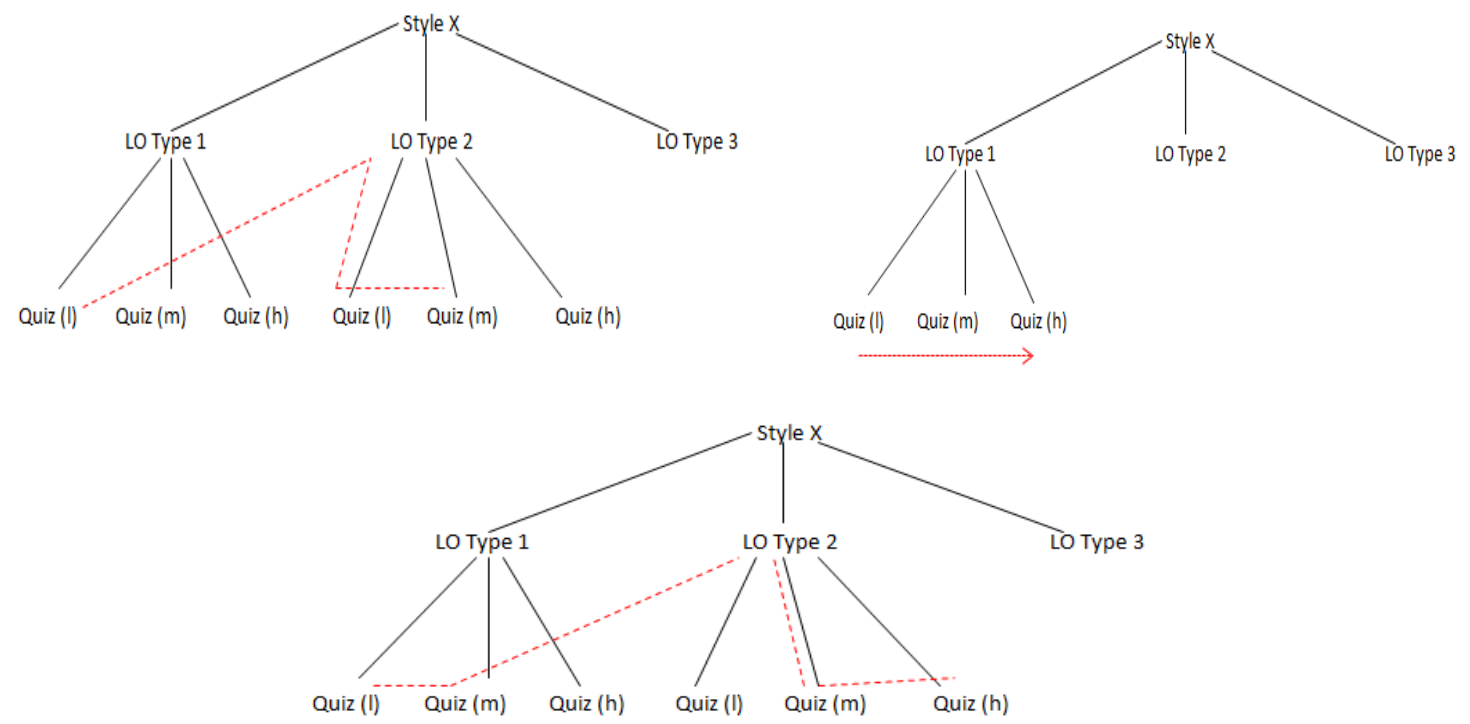

Fig. 3: Algorithm of learning personalization

These stages, for which style the activity belongs to, aimed objective and the other features to be considered for determining the learning path should be feed in to the system when the teacher uploads an activity. It is facilitated in the learning framework.

In sharable Content Object Reference Model (SCORM), (ADLS, 2011) it specifies, the lesson can be planned as sequences. Since the lesson concepts/topics are in a particular sequence composed with prerequisites, it is easy to navigate the student though such concepts based on their style. If the lesson objectives are not satisfied, the student is able to be navigated through the prerequisite path with another similar style or with same style based on their preference order until the former specified objective is satisfied. This is the idea of the Rollup option of SCORM (ADLS, 2011). In this research, I have considered the techniques of sequencing, navigation and roll up option to generate the students learning path. Different paths generated are shown in Fig. 4. The learning path is designed with prerequisites. For a particular objective, there are some targeted topics in a lesson that should be completed. Between each concepts in different lessons and within same lesson there are connections determining prerequisite knowledge. Meanwhile objectives are connected with another objectives based on prerequisites for a particular content. Initially, this was planned this model for a lesson. A course means one more level higher up. Then for adapting it for a course I have applied the same at an outer iteration level. Here what happens is, based on the way the first lessons is completed, 2nd lesson will be decided. It can be simply an inclusion or omission of a lesson for some types of lessons. For some lessons, previous lesson may indicate a level for the next lesson. However, if a lesson does not depend on a previous lesson (i.e., there is no prerequisite), then that lesson is included in the course.

Since the learner's knowledge level, dynamic and static behaviors are always observed, the styles are generated dynamically. The learning path which is structured according to the generated style at the moment is guided the student until the particular objective is satisfied. This is determined when they are ready to undertake the learning material. Each concept is evaluated individually or as a collection at the processing level and the understanding level. For such evaluations, I used formative and summative tests (Mukherjee, 2013) which are also delivered according to the individuals' style at the particular level.

\section{Evaluation and test results}

To evaluate the personalized Virtual learning environment (VLE), one of the subjects selected is 'Operating Systems'. Initially, in this subject, as a teacher, the lesson plan for the lesson called "Process Management" is prepared (Fig. 5). 


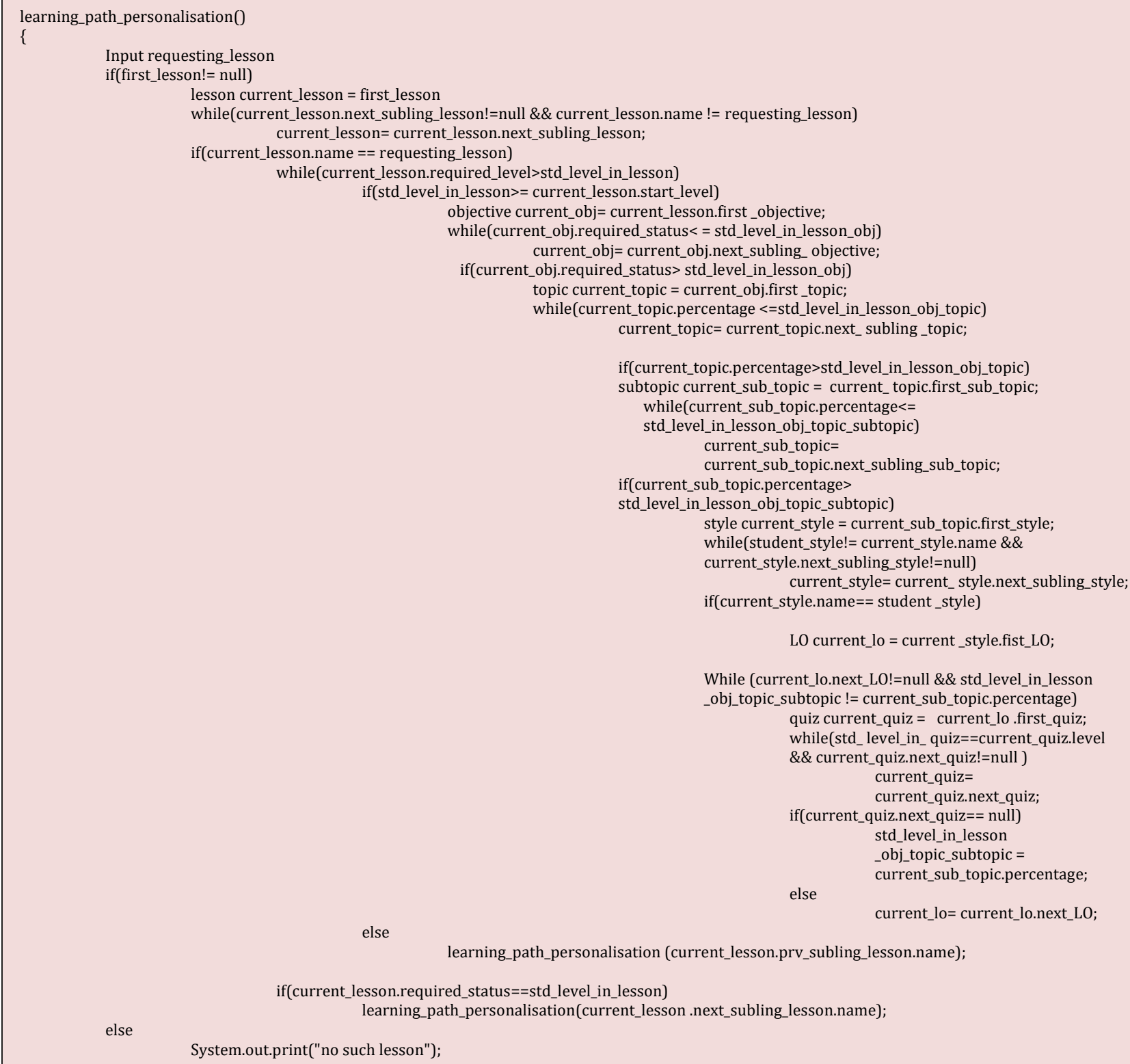

Fig. 4: Several learning paths generated

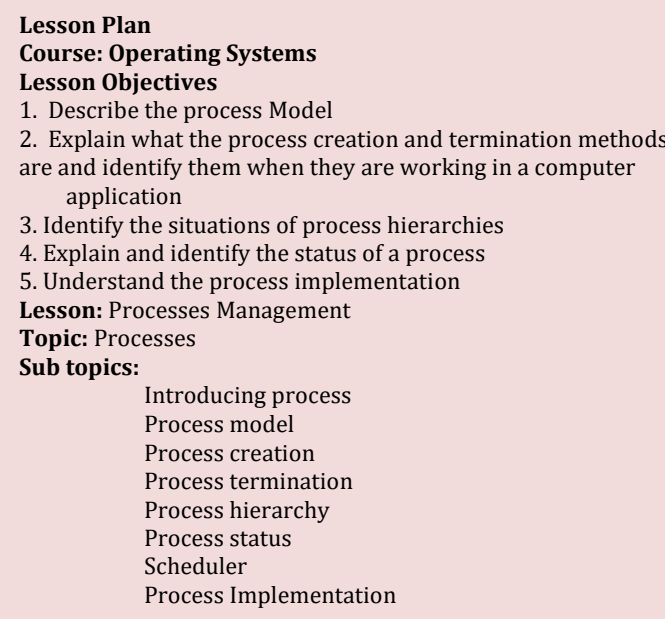

Fig. 5: Example lesson plan

Then for studied different styles of learning, different learning objects (LO) are prepared for the knowledge acquiring stage and processing stage. For each LO of each style, 3 assessments are created with different complexity.

One of the evaluations is conducted with first year first semester students who are following the degree program of Computer Science. The group contains 15 students. Throughout the lesson, they are asked to access to the VLE until they come to the standard level required. Finally a common test was conducted for all the students and performance results in Table 3 are observed. Then their average performance was compared with a performance results on the same test conducted with previous year students. The previous year the same test was conducted without this VLE. Then with another 2 tests conducted under same lesson, the performance of the students in this year is observed by allowing them to go through the VLE. Improvement of their performance is visible.

According to these results, the average performance of this year students has increased by 2.6 within the same lesson (average performance of 
previous year and this year respectively 12.6 and

15.2).

Table 3: Students marks on formative evaluation test (a) previous year (b) this year

\begin{tabular}{ccc}
\hline Std no & Marks for the quiz 1(out of 20) & Marks for the quiz 2(out of 20) \\
\hline 01 & 12 & 14 \\
02 & 18 & 20 \\
03 & 18 & 17 \\
04 & 17 & 16 \\
05 & 15 & 18 \\
06 & 12 & 15 \\
07 & 11 & 13 \\
08 & 10 & 12 \\
09 & 18 & 18 \\
10 & 18 & 20 \\
11 & 16 & 18 \\
12 & 15 & 12 \\
13 & 13 & 16 \\
14 & 17 & 16 \\
15 & 18 & 20 \\
\hline
\end{tabular}

\begin{tabular}{cc}
\hline Std no & Marks for the quiz(out of 20) \\
\hline 01 & 18 \\
02 & 15 \\
03 & 15 \\
04 & 12 \\
05 & 16 \\
06 & 11 \\
07 & 10 \\
08 & 8 \\
09 & 9 \\
10 & 7 \\
11 & 5 \\
12 & 13 \\
13 & 13 \\
14 & 18 \\
15 & 19 \\
\hline
\end{tabular}

Comparison is shown in Fig. 6. By each student, the time taken to come to the standard level was varied based on the number of paths they had to follow according to their individual knowledge level. Weak students have guided through a long learning path while the students with a better knowledge level have gone through a shorter path to reach at the expected level of the lesson. The results are obtained as follows for a 3 hours lesson. Steps are mentioned in Table 4.

With the use of application, improvement of the performance of the students in 3 lessons is calculated. When compared to the results, performance enhancement of each individual student is observed. (Individual marks of the

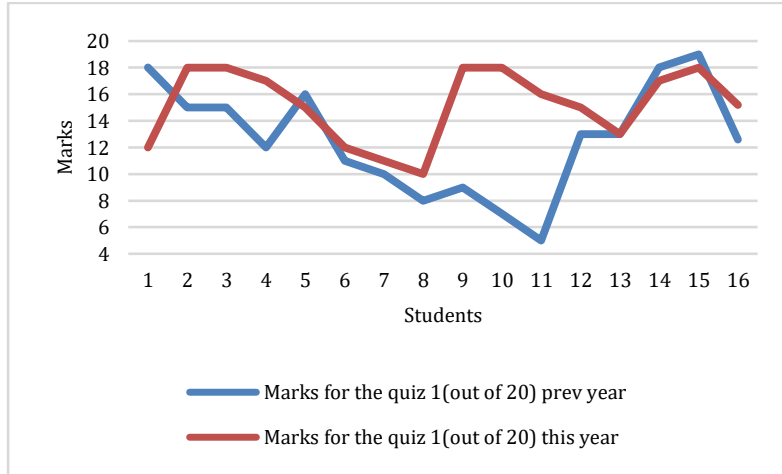

Fig. 6: Comparison of results of students in 2 years for a same test conducted with VLE and without VLE students in 3 lessons) (Fig. 7).

Table 4: Lesson paths and time taken by the students to reach at the expected knowledge level

\begin{tabular}{ccc}
\hline Std no & Time taken to reach at the expected level (hrs) & Number of paths followed to reach at the expected level \\
\hline 01 & 2 & 3 \\
02 & 2 & 3 \\
03 & 3 & 5 \\
04 & 1 & 2 \\
05 & $21 / 2$ & 4 \\
06 & 2 & 3 \\
07 & 3 & 2 \\
08 & 3 & 3 \\
09 & 3 & 3 \\
10 & 3 & 4 \\
11 & $21 / 2$ & 4 \\
12 & $13 / 4$ & 2 \\
13 & 2 & 3 \\
14 & 2 & 5 \\
15 & 2 & 5 \\
\hline
\end{tabular}

Compared to the previous year students, the area wise improvement based on the above 3 lessons are observed. A considerable amount of performance enhancement is visible according to the results set. (Average marks of each lesson of previous year students and current students)

According to the assessment marks for three lessons, the area wise average performance enhancement is visible according to the graphical representations (Table 5 and Fig. 8).

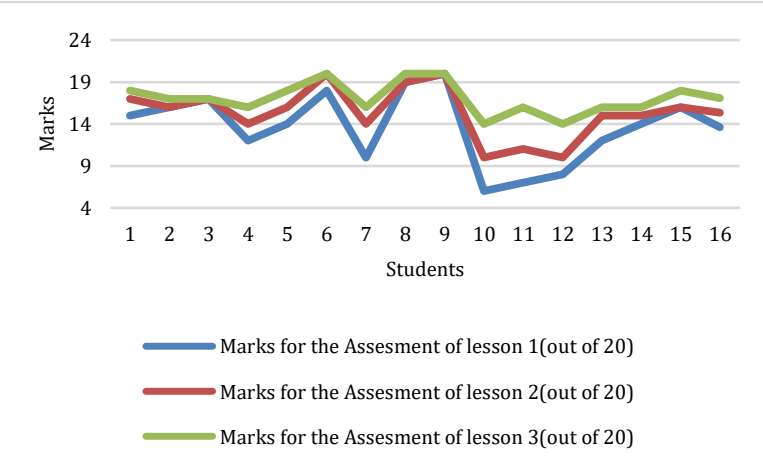

Fig. 7: Individual performance in 3 lessons 
Table 5: Area wise average performance of this year and previous year students

\begin{tabular}{ccc}
\hline Lesson & $\begin{array}{c}\text { Area wise improvement of average } \\
\text { marks of this year students }\end{array}$ & $\begin{array}{c}\text { Area wise improvement of average marks of } \\
\text { previous year students }\end{array}$ \\
\hline lesson 1 & 13.6 & 12.93333 \\
lesson 2 & 15.33333 & 13.13333 \\
lesson 3 & 17.06667 & 14.4 \\
\hline
\end{tabular}

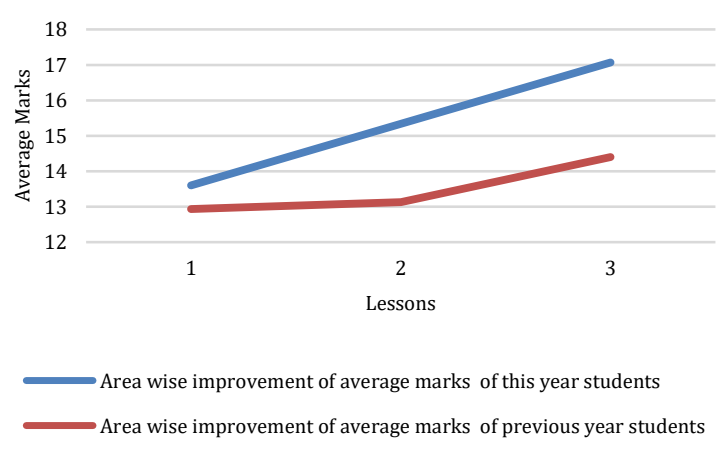

Fig. 8: Area wise average improvement of this year and previous year students

\section{Conclusion}

The main objective of this research is to emphasize the personalization of learning path leads for knowledge enhancing. This is basically a model to apply for the studies of undergraduate students. Parameters for learning path determination, which are obtained prior to the activities delivery, are the knowledge levels, dynamic and static learning behavior of the student. Each lesson is planned with the pre-determined objectives. Lesson contents are delivered at different stages with various methods according to the students' personalization parameters. From the contents, at the processing and understanding stages, the lesson objectives are evaluated. If the student is not succeeded with the objectives, they are guided through pre requisite path according to their own styles.

After the completion of the learning framework, has been exposed to undergraduate students and observed whether the learning path personalization according to the knowledge level and the style affect in knowledge enhancement. After the comparison of the delivered results with the previous year students who have been gone through the same tests without this VLE, it was able to see more than $70 \%$ of performance enhancement within the students.

\section{Acknowledgement}

This research is nearly 12 months of work effort whereby I have been accompanied and supported by many people. Here by I pay my gratitude to all of them. Especially I would like to thank all the academic and non-academic staff of Open University of Sri Lanka for guiding us throughout the entire project.

\section{References}

ADLS (2011). SCORM users guide for programmers. SCORM 2004, $4^{\text {th }}$ ed., Version 10, Advanced Distributed Learning Service, Department of Defense, Washington D.C., USA.

Bauman K and Tuzhilin A (2016). Recommending remedial learning materials to the students by filling their knowledge gaps. MIS Quarterly, 42(1): 313-332.

Graf S, Viola SR, Leo T, and Kinshuk (2007). In-depth analysis of the Felder-Silverman learning style dimensions. Journal of Research on Technology in Education, 40(1): 79-93.

Gunathilaka TMAU, Fernando MSD, and Pasqual H (2017). Identification of the learning behavior of the students for education personalization. In the International Conference on I-SMAC (IoT in Social, Mobile, Analytics and Cloud), IEEE, Palladam, India: 364-370.

Lafore R (2002). Data structures and algorithms in java. Sams Publishing, Indianapolis, USA.

McLeod SA (2013). Kolb-learning styles. Available online at: www.simplypsychology.org/learning-kolb.html

Mukherjee S (2013). Adaptive learning systems: A tool to personalize learning. Tata Interactive Systems, Mumbai, India.

Murray MC and Pérez J (2015). Informing and performing: A study comparing adaptive learning to traditional learning. Informing Science: The International Journal of an Emerging Transdicipline, 18: 111-125. 